\title{
SURFACE PHOTON EMISSIVITY OF BARE STRANGE STARS
}

\author{
K. S. CHENG ${ }^{1}$ AND T. HARKo ${ }^{1}$ \\ Received 2003 May 9; accepted 2003 June 20
}

\begin{abstract}
We consider the bremsstrahlung surface photon emissivity of strange quark stars, by systematically taking into account the effects of the multiple scatterings of highly relativistic quarks in a dense medium (the LandauPomeranchuk-Migdal effect). Because of interference between amplitudes of nearby interactions, the bremsstrahlung emissivity from a strange star surface is suppressed for frequencies smaller than a critical frequency. The range of the suppressed frequencies is a function of the quark matter density at the star's surface and of the QCD coupling constant. For temperatures much smaller than the Fermi energy of the quarks, the bremsstrahlung spectrum has the same temperature dependence as equilibrium blackbody radiation. Multiple collisions could reduce by an order of magnitude the intensity of the bremsstrahlung radiation. The effect of the thin electron layer at the surface of the quark star on the bremsstrahlung spectrum is also analyzed in detail. It is shown that absorption in the semidegenerate electron gas can also significantly reduce the intensity of the quark-quark bremsstrahlung radiation and, consequently, the surface radiation of the quark star. Hence, the combined effects of multiple collisions and absorption in the electron layer could make the soft photon surface radiation of quark stars 6 orders of magnitude smaller than equilibrium blackbody radiation.
\end{abstract}

Subject headings: dense matter — elementary particles — radiation mechanisms: nonthermal

\section{INTRODUCTION}

The quark structure of nucleons, suggested by quantum chromodynamics (QCD), opens the possibility of a hadronquark phase transition at high densities and/or temperatures, as suggested by Witten (1984). In the theories of strong interaction, quark bag models suppose that the breaking of physical vacuum takes place inside hadrons. If the hypothesis about quark matter is true, then some compact objects identified with neutron stars could actually be strange stars, built entirely of strange matter (Alcock, Farhi, \& Olinto 1986; Haensel, Zdunik, \& Schaeffer 1986). For a review of strange star properties, see Cheng, Dai, \& Lu (1998a).

Most of the investigations of quark star properties have been done within the framework of the so-called MIT bag model. Assuming that interactions of quarks and gluons are sufficiently small, neglecting quark masses, and supposing that quarks are confined to the bag volume (in the case of a bare strange star, the boundary of the bag coincides with the stellar surface), the energy density $\rho c^{2}$ and pressure $p$ of a quark-gluon plasma (QGP) are related, in the MIT bag model, by the equation of state (Cheng et al. 1998a)

$$
p=\frac{(\rho-4 B) c^{2}}{3},
$$

where $B$ is the difference between the energy density of the perturbative and nonperturbative QCD vacuum (the bag constant). Equation (1) is essentially the equation of state of a gas of massless particles with corrections due to the QCD trace anomaly and perturbative interactions.

More sophisticated investigations of quark-gluon interactions have shown that equation (1) represents a limiting case of more general equations of state. For example,

\footnotetext{
${ }^{1}$ Department of Physics, University of Hong Kong, Pokfulam Road, Hong Kong SAR, People's Republic of China; hrspksc@hkucc.hku.hk, harko@hkucc.hku.hk.
}

MIT bag models with massive strange quarks and lowest order QCD interactions lead to some correction terms in the equation of state of quark matter. Models incorporating restoration of chiral quark masses at high densities and giving absolutely stable strange matter can no longer be accurately described by using equation (1). On the other hand, in models in which quark interaction is described by an interquark potential originating from gluon exchange and by a density-dependent scalar potential that restores the chiral symmetry at high densities (Dey et al. 1998), the equation of state $P=P(\rho)$ can be well approximated by a linear function in the energy density $\rho$ (Gondek-Rosinska et al. 2000). Zdunik (2000) has studied the linear approximation of the equation of state, obtaining all parameters of the equation of state as polynomial functions of the strange quark mass, QCD coupling constant, and bag constant.

A complete description of static strange stars has been obtained based on numerical integration of mass continuity and Tolman-Oppenheimer-Volkoff (hydrostatic equilibrium) equations for different values of the bag constant (Witten 1984; Haensel et al. 1986). Using numerical methods, the maximum gravitational mass $M_{\max }$ and the maximum radius $R_{\max }$ of the strange star have been obtained, as functions of the bag constant, in the form (Witten 1984; Haensel et al. 1986; Gourgoulhon et al. 1999; Cheng \& Harko 2000; Harko \& Cheng 2002)

$$
M_{\max }=\frac{1.9638 M_{\odot}}{\sqrt{B_{60}}}, \quad R_{\max }=\frac{10.172 \mathrm{~km}}{\sqrt{B_{60}}},
$$

where $B_{60} \equiv B /\left(60 \mathrm{MeV} \mathrm{fm}^{-3}\right)$.

There are several proposed mechanisms for the formation of quark stars. Quark stars are expected to form during the collapse of the core of a massive star after a supernova explosion as a result of a first- or second-order phase transition, resulting in deconfined quark matter (Dai, Peng, \& Lu 1995). The proto-neutron star core or the neutron star core is a favorable environment for the conversion of ordinary 
matter to strange quark matter (Cheng et al. 1998b). Another possibility is that some neutron stars in low-mass $\mathrm{X}$-ray binaries can accrete sufficient mass to undergo a phase transition to become strange stars (Cheng \& Dai 1996). This mechanism has also been proposed as a source of radiation emission for cosmological $\gamma$-ray bursts (Cheng \& Dai 1998).

Strange quark matter is filled with electromagnetic waves in thermodynamic equilibrium with quarks. Photon emissivity is the basic parameter for determining the macroscopic properties of objects made of strange matter. Alcock et al. (1986) have shown that, because of very high plasma frequency near the strange matter edge, the photon emissivity of strange matter is very low. For temperatures $T \ll E_{p} / k$, where $E_{p} \approx 23 \mathrm{MeV}$ is the characteristic transverse plasmon cutoff energy, the equilibrium photon emissivity of strange matter is negligibly small, compared to that of a blackbody. The dispersion relation of the electromagnetic waves in quark matter can be written as $\omega=\left(\omega_{p}^{2}+k^{2} c^{2}\right)^{1 / 2}$, where $\omega$ is the frequency of the waves, $k$ is their wavenumber, $\omega_{p}=\left(8 \pi e^{2} c^{2} n_{b} / 3 \mu\right)^{1 / 2}$ is the plasma frequency, with $n_{b}$ the baryon number density of strange quark matter, and $\mu \approx \hbar c\left(\pi^{2} n_{b}\right)^{1 / 3}$ is the chemical potential of the quarks (Usov 1998). Propagating modes exist only for $\omega>\omega_{p}$. Hence, the lower limit of the energy $E_{\gamma}$ of the electromagnetic quanta, which are in equilibrium with the quarks, is given by $E_{\gamma}=\hbar \omega>\hbar \omega_{p} \approx 18.5\left(n_{b} / n_{0}\right)^{1 / 3} \mathrm{MeV}$, where $n_{0}=0.17 \mathrm{fm}^{-3}$ is normal nuclear matter density. At the quark star surface, $n_{b} \approx(1.5-2) n_{0}$, and the spectrum of equilibrium photons is very hard, with $\hbar \omega \approx 20-25 \mathrm{MeV}$ (Chmaj, Haensel, \& Slominski 1991). The bremsstrahlung emissivity of quark matter has been estimated by Chmaj et al. (1991). They have found that the surface radiation is about 4 orders of magnitude weaker than equilibrium blackbody radiation, even though they both have the same temperature dependence.

The structure of a realistic strange star is very complicated, but its basic properties can be described as follows (Alcock et al. 1986): Beta-equilibrated strange quark star matter consists of an approximately equal mixture of up $(u)$, down $(d)$, and strange $(s)$ quarks, with a slight deficit of the latter. The Fermi gas of $3 A$ quarks constitutes baryons of a single color, with number of baryons $A$. This structure of the quarks leads to a net positive charge inside the star. Since stars in their lowest energy state are supposed to be charge neutral, electrons must balance the net positive quark charge in strange matter stars. The electrons, being bound to the quark matter by the electromagnetic interaction and not by the strong force, are able to move freely across the quark surface but clearly cannot move to infinity because of the electrostatic attraction of the quarks. The electron distribution extends up to $\sim 10^{3} \mathrm{fm}$ above the quark surface.

The Coulomb barrier at the quark surface of a hot strange star may also be a powerful source of $e^{+} e^{-}$pairs, which are created in the extremely strong electric field of the barrier. At surface temperatures of around $10^{11} \mathrm{~K}$, the luminosity of the outflowing plasma may be of the order of $\sim 10^{51} \mathrm{ergs} \mathrm{s}^{-1}$ (Usov 1998). Moreover, as shown by Page \& Usov (2002), the thermal luminosity from the star surface, because of both photon emission and $e^{+} e^{-}$pair production, may be, for about 1 day for normal quark matter and for up to 100 yr for superconducting quark matter, orders of magnitude higher than the Eddington limit.
It is the purpose of the present paper to reconsider the problem of the photon emissivity, via bremsstrahlung radiation, of quark stars. Equilibrium radiation, transmitted through the surface, is dominant at temperatures $T>2 \times 10^{10} \mathrm{~K}$, while below this temperature the bremsstrahlung radiation from the surface layer prevails. Hence, bremsstrahlung is the main source of radiation for cold quark stars. This electromagnetic radiation is generated in the dense medium at the stellar surface in which extremely relativistic quarks move. In the 1950s Landau \& Pomeranchuk (1953) predicted that the cross section for bremsstrahlung from highly relativistic particles in dense media is suppressed, because of the interference between amplitudes of nearby interactions. The suppression has its roots in the uncertainty principle. The kinematics of bremsstrahlung require that the longitudinal momentum transfer between the fixed and the scattered particles must be small. On the other hand, the uncertainty principle requires that the interaction must occur over a large longitudinal distance scale (formation zone). If a charged particle Coulomb scatters while traversing this zone, the bremsstrahlung amplitude from before and after the scattering can interfere, reducing the amplitude for photon emission (Landau \& Pomeranchuk 1953; Anthony et al. 1995; Klein 1999). This effect has been recently confirmed experimentally for the bremsstrahlung emission of electrons in different materials at a 5\% accuracy level (Anthony et al. 1995). Since the surface of the quark star consists of a very dense medium, with density of the order of $4 B \approx 4 \times 10^{14}$, multiple collisions of electromagnetically radiating particles lead to a significant suppression of the quark-quark bremsstrahlung. By adopting a simple model for the elastic scattering of quarks, we derive the spectrum of classical bremsstrahlung radiation and the emissivity of the quark matter, by considering the multiple scattering effects in the electromagnetic radiation of quark matter. For the range of densities existing at the surfaces of strange stars, which are higher than the nuclear density, the Landau-Pomeranchuk-Migdal effect could reduce the bremsstrahlung emissivity of quark matter by an order of magnitude.

Photon emissivity of the QGP, which is conjectured to be formed in ultrarelativistic heavy-ion collisions, has been extensively investigated recently (for a recent review of direct photon emission from QGP, including comparisons of theoretical predictions with experiments, see Peitzmann \& Thoma 2002). Photons and dilepton pairs only interact electromagnetically, and their mean free paths are much larger than the size of the QGP. Hence, these electromagnetic probes leave the hot and dense QGP without further scattering, thus providing important information about the early stages of the collision and structure of the QGP. As sources of direct photons, one can consider quark annihilation, Compton scattering, and bremsstrahlung following the initial hard scattering of partons of the nuclei, as well as thermal photons from the QGP and from hadronic interactions in the hot hadronic gas after the hadronization of the plasma (Peitzmann \& Thoma 2002). The first observation of direct photon production in ultrarelativistic heavy-ion collisions has been reported by the WA98 collaboration in ${ }^{208} \mathrm{~Pb}+{ }^{208} \mathrm{~Pb}$ collisions at $\sqrt{s}=158 \mathrm{GeV}$ in the Super Proton Synchrotron at CERN (Aggarwal et al. 2000). The results display a clear excess of direct photons above the expected background from hadronic decays in the range of transverse momentum $p_{T}>1.5 \mathrm{GeV} c^{-1}$ in the most central 
collisions. These experimental findings provide a confirmation of the feasibility of direct photons as reliable probes in heavy-ion collisions and may pave the way for the understanding of the formation and evolution of the QGP.

The evaluation of the photoemission rate from the QGP was initiated by Kapusta, Lichard, \& Seibert (1991) and by Baier et al. (1992). However, as shown by Aurenche, Gelis, \& Zaraket (2000), these initial estimations were incomplete, since the bremsstrahlung and inelastic pair annihilation processes contain collinear enhancements that cause them to contribute at the same parametric order in the coupling as the two-to-two processes, even for large photon energies. However, these calculations are also incomplete, since they do not incorporate the suppression of photon emission due to multiple scatterings during the photon emission process, which limits the coherence length of the emitted radiation (the Landau-Pomeranchuk-Migdal [LPM] effect). The rate of photoproduction by bremsstrahlung and inelastic pair annihilation in a hot, equilibrated plasma at zero chemical potential, fully including the LPM effect, was calculated to leading orders in both the electromagnetic and strong coupling constants by Arnold, Moore, \& Yaffe $(2001,2002)$. The emission of hard photons from the QGP plasma, using a model based on the thermodynamics of QCD, and the determination of the initial temperature of the expanding fireball has been considered recently by Renk (2003).

The radiation emitted by the quark layer at the surface of the quark star must also cross the thin electron layer extending beyond the star's surface. We also consider the effect of this layer on the outgoing radiation and show that it can significantly reduce the intensity of the radiation coming from the quark star.

The present paper is organized as follows: In $\S 2$ we discuss the LPM effect for quark matter. The bremsstrahlung emissivity of quark matter is considered in $\S 3$. The effect of the electron layer on the radiation outgoing from the quark star surface is analyzed in $\S 4$. In $\S 5$ we discuss and conclude our results. Throughout this paper we use units so that $c=\hbar=k_{\mathrm{B}}=1$, with $k_{\mathrm{B}}$ the Boltzmann constant.

\section{LANDAU-POMERANCHUK-MIGDAL EFFECT IN QUARK MATTER}

The dispersion relation shows that only photons with frequency $\omega>\omega_{p}$ can propagate inside strange matter. The propagation of electromagnetic waves of frequency lower than $\omega_{p}$ is exponentially damped, with the damping coefficient $\beta$ depending on $\omega$ and $\omega_{p}$. For $\omega \ll \omega_{p}$, the value of $\beta$ is given by $\beta \approx 2 \omega_{p}$ (Jackson 1999). This means that photons with $\omega<\omega_{p}$ emitted in various processes inside strange matter are absorbed. The mean free path of such a photon is of the order of $\lambda \approx c / \beta \approx c / 2 \omega_{p} \approx 5 \mathrm{fm}$ (Chmaj et al. 1991). Therefore, only photons produced just below the surface with momenta pointing outward can leave strange matter.

In the following we consider the photon emissivity of quark matter by also taking into account the effect of multiple scatterings on the emitted radiation. This effect is called the LPM effect and was considered initially by Landau \& Pomeranchuk (1953), who used classical electrodynamics to demonstrate bremsstrahlung suppression due to multiple scattering: the suppression comes from the interference between photons emitted by different elements of the charged particle trajectory. To study the astrophysical implications of this effect on quark matter, we take into account the bremsstrahlung photons produced in the quark-quark interactions, that is, in the process $q_{1}+q_{2} \rightarrow$ $q_{1^{\prime}}+q_{2^{\prime}}+\gamma$.

The LPM effect can be discussed in a simple qualitative way as follows: Let us consider a bremsstrahlung photon of energy $\omega$ produced by an ultrarelativistic quark of energy $E$ and mass $m_{\mathrm{eff}}$, where $\omega \ll E$. In this kinematic regime, the average angle $\theta_{\gamma}$ between the incident quark and the produced photon is small, $\theta_{\gamma} \approx m_{\mathrm{eff}} / E$. We assume that the angle between the incident and scattered quark is also small. Moreover, we assume that all quarks have the same density-dependent effective mass $m_{\text {eff }} \approx \mu\left(4 \alpha_{s} / 3 \pi\right)^{1 / 2} \approx$ $\left(4 \alpha_{s} / 3 \pi\right)^{1 / 2}\left(\pi^{2} n_{b}\right)^{1 / 3}$, where $\alpha_{s}$ is the QCD coupling constant and $n_{b}=\left(n_{u}+n_{d}+n_{s}\right) / 3$, with $n_{j}, j=u, d, s, \ldots$, being the quark particle number densities. Neglecting the scattering and quark-photon angles, it follows that the longitudinal momentum transfer $p_{\|}$to the static quark is $p_{\|} \approx \omega / 2 \gamma^{2}$, where $\gamma=E / m_{\text {eff }}$ (Klein 1999). The uncertainty principle then requires that the spatial position of the bremsstrahlung process have a longitudinal uncertainty of $L_{\|} \sim 1 / p_{\|} \approx 2 \gamma^{2} / \omega$. In alternate language, the quark and photon slowly split apart over the distance $L_{\|}$. In a sufficiently dense medium, the quark mean free path is much less than $L_{\|}$; in the LPM effect, the relevant interaction is multiple scattering.

Bremsstrahlung is suppressed when the mean square multiple scattering angle over the distance $L_{\|}, \theta_{\mathrm{ms}}^{2}=$ $\left(E_{S} / E\right)^{2}\left(L_{\|} / X_{0}\right)$, is greater than or equal to $\theta_{\gamma}^{2}$, where $E_{s}=m_{\mathrm{eff}}(4 \pi / \alpha)^{1 / 2}, \quad X_{0}=\left[4 n \alpha r_{q}^{2} Z^{2} \ln \left(184 Z^{-1 / 3}\right)\right]^{-1}, \quad$ and $r_{q}=e^{2} / m_{\mathrm{eff}}$ is the radiation length (Anthony et al. 1995; Klein 1999). Therefore, the bremsstrahlung differential cross section for the production of a photon is suppressed when $\omega<\omega_{\mathrm{LPM}}=E^{2} / E_{\mathrm{LPM}}$, where $E_{\mathrm{LPM}}=m_{\mathrm{eff}}^{2} X_{0} \alpha / 8 \pi$ (Anthony et al. 1995; Klein 1999). By assuming that the temperature of the star $T \ll p_{\mathrm{F}}$, with $p_{\mathrm{F}}$ the Fermi momentum, the Fermi distribution factors force all the quarks to have $E \approx \mu$. For an extremely relativistic quark gas, $E \approx p_{\mathrm{F}}=\left(6 \pi^{2} n_{b} / g\right)^{1 / 3}$, where $g$ is the statistical weight for quark matter. Therefore, the critical LPM frequency for bremsstrahlung photon emission in quark matter is given by

$$
\omega_{\mathrm{LPM}}=\frac{2^{1 / 3} 3^{4 / 3} \pi^{5 / 3} g^{2 / 3} e^{4} Z^{2} \ln \left(184 Z^{-1 / 3}\right)}{\alpha_{s}^{2}} n_{b}^{1 / 3}=a n_{b}^{1 / 3},
$$

where

$$
a=\frac{2^{1 / 3} 3^{4 / 3} \pi^{5 / 3} g^{2 / 3} e^{4} Z^{2} \ln \left(184 Z^{-1 / 3}\right)}{\alpha_{s}^{2}} .
$$

For strange quark matter, $g=6$.

Emission of the bremsstrahlung radiation with frequency smaller than $\omega_{\text {LPM }}$ is suppressed, because of the effect of multiple scattering. As expected, the LPM frequency increases with the density of the strange matter. The variation of $\omega_{\text {LPM }}$ as a function of the particle number density is represented, for different values of the strong coupling constant $\alpha_{s}$, in Figure 1.

The most important parameter characterizing the LPM effect is the average time between two collisions, denoted $\tau$. It can be calculated from $\tau^{-1}=n \sigma_{0} v$, where $n$ is the density of the medium, which depends on its composition and temperature (Cleymans, Goloviznin, \& Redlich 1993). Here, $\sigma_{0}$ denotes the cross section of a test particle with velocity $v$ 


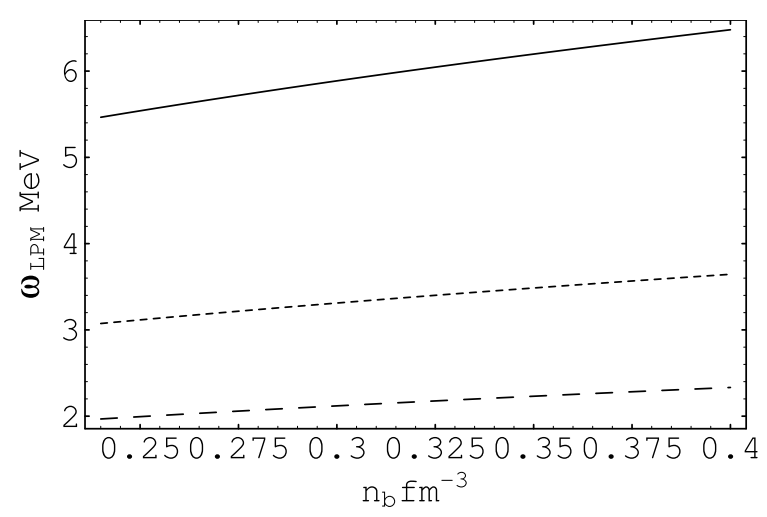

FIG. 1.-Variation, as a function of the quark number density $n_{b}$, of the LPM frequency $\omega_{\text {LPM }}$ for different values of the strong coupling constant: $\alpha_{s}=0.3$ (solid curve), 0.4 (dotted curve), and 0.5 (dashed curve).

and interacting with the particles of the medium. Thus, generally, $\tau$ is a function of the temperature and density of the medium and of the momentum of the test particle. The value of $\tau$ is essential to the importance of the LPM effect: if $\tau^{-1}$ is $0(\tau \rightarrow \infty)$, the effect is absent (Cleymans et al. 1993).

The starting point for the rigorous implementation of the LPM effect is the following textbook equation for the energy radiated per unit momentum by a charged particle (Jackson 1999):

$$
\frac{d I}{d^{3} k}=\frac{\alpha}{(2 \pi)^{2}} \sum_{l=u, d, s}\left(\frac{q_{l}}{e}\right)^{2}\left|\int_{-\infty}^{+\infty} e^{i[\omega t-\boldsymbol{k} \cdot \boldsymbol{r}(t)]} \boldsymbol{n} \times \boldsymbol{v}_{l} d t\right|^{2},
$$

where $\boldsymbol{r}_{l}(t)$ describes the trajectory of the charged particle, $\boldsymbol{v}_{l}(t)$ is its velocity, $\boldsymbol{n}=\boldsymbol{k} /|\boldsymbol{k}|, \alpha$ is the fine-structure constant, and $q_{l}$ is the charge of the $(u, d, s)$ quarks. If only one collision occurs and if the velocity is a constant $\left(\boldsymbol{v}=\boldsymbol{v}_{1}\right)$ before and after the collision $\left(\boldsymbol{v}=\boldsymbol{v}_{2}\right)$, then the radiated energy is given by (Jackson 1999)

$$
\begin{aligned}
\frac{d I}{d^{3} k}= & \frac{\alpha}{(2 \pi)^{2}} \sum_{l=u, d, s}\left(\frac{q_{l}}{e}\right)^{2} \mid \int_{-\infty}^{t_{1}} e^{i\left(\omega-\boldsymbol{k} \cdot \boldsymbol{v}_{11)} t\right.} \boldsymbol{n} \times \boldsymbol{v}_{1 l} d t \\
& \quad+\int_{-\infty}^{t_{1}} e^{i\left(\omega-\boldsymbol{k} \cdot \boldsymbol{v}_{2 l}\right) t} \boldsymbol{n} \times\left.\boldsymbol{v}_{2 l} d t\right|^{2} \\
= & \frac{\alpha}{(2 \pi)^{2}} \sum_{l=u, d, s}\left(\frac{q_{l}}{e}\right)^{2}\left|\frac{\boldsymbol{n} \times \boldsymbol{v}_{1 l}}{\omega-\boldsymbol{k} \cdot \boldsymbol{v}_{1 l}}+\frac{\boldsymbol{n} \times \boldsymbol{v}_{2 l}}{\omega-\boldsymbol{k} \cdot \boldsymbol{v}_{2 l}}\right|^{2}
\end{aligned}
$$

which is the standard textbook form for the calculation of the intensity.

If, however, a series of collisions occurs, one has to change equations (4) and (5) to a sum over all pieces of the trajectory (Cleymans et al. 1993):

$$
\begin{aligned}
\frac{d I}{d^{3} k}=\frac{\alpha}{(2 \pi)^{2}} \sum_{l=u, d, s}\left(\frac{q_{l}}{e}\right)^{2} \mid \int_{-\infty}^{t_{1}} e^{i\left[\omega t-\boldsymbol{k} \cdot \boldsymbol{r}_{l}(t)\right]} \boldsymbol{n} \times \boldsymbol{v}_{1 l} d t \\
+\int_{t_{1}}^{t_{2}} e^{i\left[\omega t-\boldsymbol{k} \cdot \boldsymbol{r}_{l}(t)\right]} \boldsymbol{n} \times \boldsymbol{v}_{2 l} d t+\ldots \\
+\int_{t_{n-1}}^{t_{n}} e^{i\left[\omega t-\boldsymbol{k} \cdot \boldsymbol{r}_{l}(t)\right]} \boldsymbol{n} \times \boldsymbol{v}_{n l} d t+\left.\ldots\right|^{2} .
\end{aligned}
$$

Assuming that the velocity is a constant between two collisions leads to

$$
\begin{aligned}
\frac{d I}{d^{3} k}=\frac{\alpha}{(2 \pi)^{2}} \sum_{l=u, d, s}\left(\frac{q_{l}}{e}\right)^{2} & \mid \sum_{j=1}^{N} e^{i\left(\omega t_{j-1 l}-\boldsymbol{k} \cdot \boldsymbol{r}_{j-1 l}\right)} \\
& \times\left.\left(1-e^{-i\left(\omega-\boldsymbol{k} \cdot \boldsymbol{v}_{j l}\right)\left(t_{j}-t_{j-1}\right)}\right)\right|^{2},
\end{aligned}
$$

where $N$ is the total number of collisions. The calculation of the square gives

$$
\begin{aligned}
\frac{d I}{d^{3} k}= & \frac{\alpha}{(2 \pi)^{2}} \sum_{l=u, d, s}\left(\frac{q_{l}}{e}\right)^{2} \sum_{j=1}^{N} \frac{\boldsymbol{v}_{j l}^{2}-\left(\boldsymbol{n} \cdot \boldsymbol{v}_{j l}\right)^{2}}{\left(\omega-\boldsymbol{k} \cdot \boldsymbol{v}_{j l}\right)^{2}} \\
& \times\left(2-e^{i \xi_{j}\left(\omega-\boldsymbol{k} \cdot \boldsymbol{v}_{j l}\right)}-e^{-i \xi_{j}\left(\omega-\boldsymbol{k} \cdot \boldsymbol{v}_{j l}\right)}\right) \\
& +2 \frac{\alpha}{(2 \pi)^{2}} \sum_{m=u, d, s}\left(\frac{q_{m}}{e}\right)^{2} \\
& \times \operatorname{Re} \sum_{j>l}^{N} \frac{\boldsymbol{v}_{j m} \cdot \boldsymbol{v}_{l m}-\left(\boldsymbol{n} \cdot \boldsymbol{v}_{j m}\right)\left(\boldsymbol{n} \cdot \boldsymbol{v}_{l m}\right)}{\left(\omega-\boldsymbol{k} \cdot \boldsymbol{v}_{j m}\right)\left(\omega-\boldsymbol{k} \cdot \boldsymbol{v}_{l m}\right)} \\
& \times \exp \left[i \sum_{i=l}^{j-1} \xi_{i}\left(\omega-\boldsymbol{k} \cdot \boldsymbol{v}_{i m}\right)\right] \\
& \times\left(1-e^{-i\left(\omega-\boldsymbol{k} \cdot \boldsymbol{v}_{j m}\right) \xi_{j}}\right)\left(1-e^{-i\left(\omega-\boldsymbol{k} \cdot \boldsymbol{v}_{l m}\right) \xi_{l}}\right),
\end{aligned}
$$

where we denoted $\xi_{j}=t_{j}-t_{j-1}$.

Since the quark gas is taken to be in thermal equilibrium, many possible velocities can result in between two collisions. For this reason, the nondiagonal terms in the above equation will give zero contribution after one averages over all velocities, providing of course that no correlation exists between the velocity before and after the collision. Only the diagonal terms remain in this case. The time between two successive collisions is given by $\xi$. Taking an average over the time between two collisions can be obtained using the following distribution (Cleymans et al. 1993)

$$
\frac{d W}{d \xi}=\frac{1}{\tau} e^{-\xi / \tau} .
$$

Then, the electromagnetic energy radiated away by the quarks is given by

$$
\begin{aligned}
\frac{d I}{d^{3} k}= & \frac{\alpha}{(2 \pi)^{2}} \sum_{l=u, d, s}\left(\frac{q_{l}}{e}\right)^{2} \frac{1}{\tau} \int_{0}^{\infty} e^{-\xi \tau^{-1}} \frac{v_{l}^{2}-\left(\boldsymbol{n} \cdot \boldsymbol{v}_{l}\right)^{2}}{\left(\omega-\boldsymbol{k} \cdot \boldsymbol{v}_{l}\right)^{2}} \\
& \times\left(2-e^{i\left(\omega-\boldsymbol{k} \cdot \boldsymbol{v}_{l}\right) \xi}-e^{-i\left(\omega-\boldsymbol{k} \cdot \boldsymbol{v}_{l}\right) \xi}\right) d \xi,
\end{aligned}
$$

where we have introduced the average velocities by means of the definitions $\boldsymbol{v}=\langle\boldsymbol{v}\rangle=\sum_{j=1}^{N} \boldsymbol{v}_{j} / N, \boldsymbol{v}^{2}=\left\langle\boldsymbol{v}^{2}\right\rangle=$ $\sum_{j=1}^{N} \boldsymbol{v}_{j}^{2} / N$, and we have also denoted $v=|\langle\boldsymbol{v}\rangle|$.

The averaging over the time between two collisions can be done with the use of the mathematical identity $a \int_{0}^{\infty}(1-\cos b \xi) e^{-a \xi} d \xi=b^{2} /\left(a^{2}+b^{2}\right)$. Therefore, we obtain for the intensity of the radiation emitted by a quark moving in a dense medium the expression

$$
\frac{d I}{d^{3} k}=\frac{\alpha}{2 \pi^{2}} \sum_{l=u, d, s}\left(\frac{q_{l}}{e}\right)^{2} \frac{v_{l}^{2}-\left(\boldsymbol{n} \cdot \boldsymbol{v}_{l}\right)^{2}}{\tau^{-2}+(\omega-\boldsymbol{k} \cdot \boldsymbol{v})^{2}} .
$$


Since $d^{3} k=\omega^{2} d \omega d \Omega$, the intensity can also be written as

$$
d I=\frac{\alpha}{2 \pi^{2}} \sum_{l=u, d, s}\left(\frac{q_{l}}{e}\right)^{2} v_{l}^{2} \frac{1-\cos ^{2} \theta}{\tau^{-2}+\left(\omega-k v_{l} \cos \theta\right)^{2}} \omega^{2} d \omega d \Omega .
$$

The angular integration is standard, and finally we obtain for the intensity of the radiation emitted by a quark moving in a dense medium the expression (Cleymans et al. 1993)

$$
d I=\frac{\alpha}{\pi} \sum_{l=u, d, s}\left(\frac{q_{l}}{e}\right)^{2} \frac{1}{v_{l}} \Phi\left(\omega, v_{l}, \tau^{-1}\right) \frac{d \omega}{\omega},
$$

where the function $\Phi\left(\omega, v, \tau^{-1}\right)$ is given by

$$
\begin{aligned}
\Phi\left(\omega, v_{l}, \tau^{-1}\right)= & \frac{1-\tau^{2} \omega^{2}\left(1-v_{l}^{2}\right)}{\tau} \\
& \times\left[\arctan \tau \omega\left(1+v_{l}\right)-\arctan \tau \omega\left(1-v_{l}\right)\right] \\
& +\omega \ln \frac{1+\omega^{2} \tau^{2}\left(1+v_{l}\right)^{2}}{1+\omega^{2} \tau^{2}\left(1-v_{l}\right)^{2}}-2 \omega v_{l} .
\end{aligned}
$$

In order to compute the numerical values of the intensity, we need to know the mean velocities of the quarks randomly moving in a dense medium. The multiple scatterings of the quarks change the quark path and affect the emission of the radiation. If $\boldsymbol{v}(0)$ is the initial velocity of the quark at the moment $t=0$, then at the moment $t$ its velocity will be

$$
\boldsymbol{v}(t)=\boldsymbol{v}_{z}(t)+\boldsymbol{v}_{\perp}(t)=\boldsymbol{v}(0)\left[1-\theta_{\mathrm{ms}}^{2}(t) / 2\right]+|\boldsymbol{v}(0)| \theta_{\mathrm{ms}}(t) \boldsymbol{\Theta},
$$

where $\Theta$ is a unit vector perpendicular to the initial direction of motion and $\theta_{\mathrm{ms}}(t)$ is the electron multiple scattering in the time interval 0 to $t$ (Klein 1999). Landau \& Pomeranchuk (1953) took $\theta_{\mathrm{ms}}^{2} \approx\left\langle\theta_{\mathrm{ms}}^{2}\right\rangle$. Therefore, in this approximation we obtain $v=v(0)\left(1+\left\langle\theta_{\mathrm{ms}}^{2}\right\rangle^{2} / 4\right)^{1 / 2}$. Over a distance $\boldsymbol{d}=\boldsymbol{v}_{z} t$, the multiple scattering is given by $\left\langle\theta_{\mathrm{ms}}^{2}\right\rangle=\left(E_{s} / E\right)^{2}\left(d / X_{0}\right)$. For $d$ we assume that it is the suppression distance, that is, the distance over the interaction actually spread: $d=l_{f}$. In the most general case, with quantum corrections taken into account, $l_{f}$ is given as a root of a quadratic equation: $l_{f}=l_{f 0}\left(1+E_{s}^{2} l_{f} / 2 m_{\mathrm{eff}}^{2} X_{0}\right)^{-1}$, where $l_{f 0}=2 E^{2} / \omega m_{\mathrm{eff}}^{2}$ is the classical suppression length (Klein 1999).

In the case of the standard approach to the bremsstrahlung radiation of charged particles, one assumes that the energy is emitted in a single collision. Then, from equation (5), by taking $\boldsymbol{v}_{2}=0$ and $\boldsymbol{v}_{1}=\boldsymbol{v}$, it immediately follows that the single-collision spectral distribution of the bremsstrahlung radiation $d I_{\mathrm{sc}}$ is given by (Landau \& Lifshitz 1975)

$$
d I_{\mathrm{sc}}=\frac{\alpha}{\pi} \sum_{l=u, d, s}\left(\frac{q_{l}}{e}\right)^{2}\left(\frac{1}{v_{l}} \ln \frac{1+v_{l}}{1-v_{l}}-2\right) d \omega .
$$

The total intensity is proportional to the frequency of the emitted radiation and is independent of the medium in which the motion of the particle takes place. The same result can be derived by taking the limit $\tau \rightarrow \infty$ in equation (13):

$$
\frac{d I_{\mathrm{sc}}}{d \omega}=\lim _{\tau \rightarrow \infty} \frac{d I}{d \omega} .
$$

In the opposite limit of very high density, corresponding to $\tau \rightarrow 0$, from equation (13) we obtain

$$
\lim _{\tau \rightarrow 0} \frac{d I}{d \omega}=0
$$

and $\lim _{\tau \rightarrow 0} I=0$. Therefore, the electromagnetic radiation from a quark moving in a very dense medium is completely suppressed.

\section{BREMSSTRAHLUNG EMISSIVITY OF STRANGE STARS}

To calculate the bremsstrahlung emissivity, a sensible model for quark interactions has to be used. We adopt the basic assumption that the details of the interaction should be relatively unimportant, as long as the overall strength is correct and the relevant symmetries are respected. Hence, we use the well-known Nambu-Jona-Lasinio (NJL) model in its $S U(2)$ version in our calculations (Hatsuda \& Kunihiro 1994). It has the same symmetries as QCD and describes an effective pointlike interaction with a constant coupling strength. Usually, the NJL model and its extensions are used in mean field calculations. As a consequence of adopting this model, all the aspects related to color superconductivity are neglected.

Let $d \sigma_{0}$ be the cross section for a given process of scattering of charged particles, which may be accompanied by the emission of a certain number of photons. For example, $d \sigma_{0}$ could refer to the scattering of a quark by another quark, with the possible emission of hard photons. Together with this process, one could consider another process that differs from it only in that one extra photon is emitted. In this case the total cross section $d \sigma$ can be represented as a product of two independent factors, the cross section $d \sigma_{0}$ and the probability $d W_{\gamma}$ of emission of a single photon in the collision (Berestetskii, Lifshitz, \& Pitaevskii 1982). The emission of a soft photon is a quasi-classical process. The probability of emission is the same as the classically calculated number of quanta emitted in the collision, that is, the same as the classical intensity (total energy) of the emission $d I$ divided by the frequency of the radiation $\omega$ (Berestetskii et al. 1982). Hence, as a first step in obtaining the bremsstrahlung emissivity of quark matter, we have to calculate the classical radiation intensity emitted by a quark moving in a dense medium in which many interparticle collisions occur.

The probability $d W_{\gamma}$ of emitting a photon of energy $\omega$ by any of the scattered quarks is $d W_{\gamma}=d I / \omega$. Therefore, the total cross section for the emission of soft bremsstrahlung photons is given by (Berestetskii et al. 1982)

$$
d \sigma=d \sigma_{0} \frac{d I}{\omega} .
$$

To calculate the total cross section for a photon emission, we need to know $d \sigma_{0}$, describing the scattering of quarks. Within the two-flavor NJL model, the elementary quarkquark cross sections have been calculated by Zhuang et al. (1995). The calculations have been done nonperturbatively in the coupling constant, using the so-called $1 / N_{c}$ expansion, where $N_{c}$ is the number of colors. In the first order of the $1 / N_{c}$ expansion and for temperature $T<T_{c}$, where $T_{c}=0.19 \mathrm{GeV}$, the quark-quark scattering cross sections have a remarkably simple dependence on the collision energy $s$, given by $\sigma_{0}(s) \sim 1 / s$ (Zhuang et al. 1995). The 
cross section decreases with increasing energy. For a more general parameterization of the quark-quark scattering cross section, with the inclusion of the double-scattering terms, see also Manley (1997).

We assume that the quarks form a degenerate Fermi gas, with the particle number density $n$ given by

$$
\begin{aligned}
n & =\frac{g}{2 \pi^{2}} \int_{0}^{\infty} f[E(p)-\mu] p^{2} d p \\
& =\frac{g}{2 \pi^{2}} \int_{0}^{\infty} \frac{p^{2} d p}{\exp \{[E(p)-\mu] / T\}+1},
\end{aligned}
$$

where $E=\left(p^{2}+m^{2}\right)^{1 / 2}$ is the quark kinetic energy and $f([E(p)-\mu]=1 /(\exp \{[E(p)-\mu] / T\}+1)$ is the FermiDirac distribution function (Chiu 1968).

In order to estimate the importance of the LPM effect on the bremsstrahlung emissivity of quark matter, we have to estimate $\tau$, the average time between two collisions. In the case of the interaction of two quarks, denoted 1 and 2, the average time can be obtained from (Haglin \& Pratt 1994)

$$
\tau^{-1}=\int d s \frac{d^{3} p_{2}}{(2 \pi)^{3}} f\left(p_{2}\right) \sigma_{12}(s) v_{\text {rel }} \delta\left[s-\left(p_{1}+p_{2}\right)^{2}\right],
$$

where

$$
\begin{aligned}
v_{\text {rel }} & =\left|\boldsymbol{v}_{1}-\boldsymbol{v}_{2}\right|=\frac{\sqrt{\left(p_{1} \cdot p_{2}\right)^{2}-m_{1}^{2} m_{2}^{2}}}{E_{1} E_{2}} \\
& =\frac{\sqrt{s\left(s-4 m_{\mathrm{eff}}^{2}\right)}}{2 E_{1} E_{2}} .
\end{aligned}
$$

The kinematic invariant $s$ is given by $s=$ $2\left(m_{\text {eff }}^{2}+E_{1} E_{2}-p_{1} p_{2} \cos \theta\right)$ (Cleymans et al. 1993). By assuming that quarks are ultrarelativistic, with $E=p$, the average collision time can be approximated by the following analytic representation:

$$
\tau^{-1} \approx-\frac{1}{8 \pi^{8 / 3}} n_{b}^{-1 / 3} T^{2} \operatorname{Li}_{2}\left\{-\exp \left[\frac{\left(\pi^{2} n_{b}\right)^{1 / 3}}{T}\right]\right\},
$$

where $\operatorname{Li}_{2}(z)$ is the polylogarithmic function defined as $\mathrm{Li}_{n}(z)=\sum_{k=1}^{\infty} z^{k} / k^{n}$. The variation of the collision time as a function of the quark number density is presented, for different values of the temperature, in Figure 2.

In the framework of our model and for the range of temperatures considered, the collision time is almost independent of $T$. As expected, $\tau$ decreases with increasing density. To calculate $\tau$ we have assumed again that the test quarks, moving in the dense medium, have energies of the same order as the Fermi energy, $E_{1} \approx E_{\mathrm{F}} \approx p_{\mathrm{F}} \approx\left(\pi^{2} n_{b}\right)^{1 / 3}$. A rough estimate of the mean collision time can also be obtained from the relation $\tau=1 / n \sigma_{0} v$. By assuming that the cross section of quark-quark interactions is around $\sigma_{0} \approx 1$ mbarn (Manley 1997) and that the quarks are traveling with the speed of light, we obtain $\tau=41.6 \mathrm{fm}$ $\left(=12.48 \times 10^{-23} \mathrm{~s}\right)$ for $n_{b}=0.24 \mathrm{fm}^{-3}$ and $\tau=25 \mathrm{fm}$ $\left(=7.5 \times 10^{-23} \mathrm{~s}\right)$ for $n_{b}=0.4 \mathrm{fm}^{-3}$.

The knowledge of $\tau$ allows the calculation of the spectrum of the photons emitted via the bremsstrahlung mechanism from the quark star surface. The applicability of the formulae for the spectral distribution and intensity of the bremsstrahlung radiation that we derived in $\S 2$ is limited

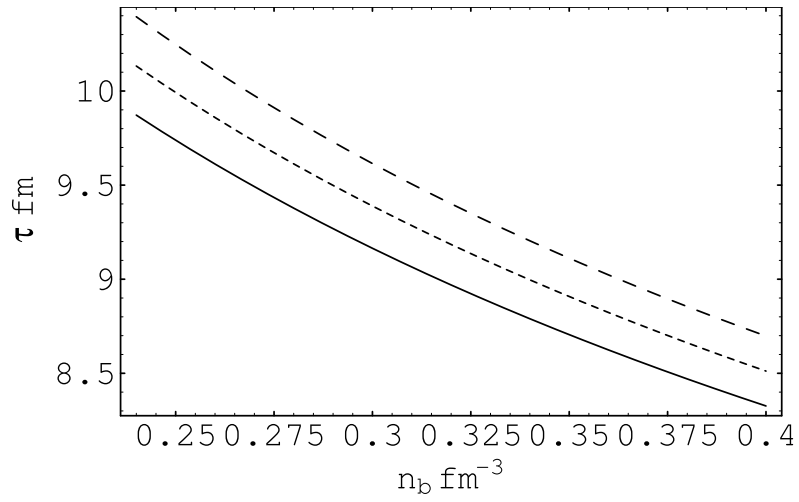

FIG. 2.-Variation, as a function of the quark number density, of the mean collision time $\tau$ for different values of the temperature of the surface of the strange star: $T=0.10$ (solid curve), 5 (dotted curve), and $10 \mathrm{MeV}$ (dashed curve).

by the quantum condition that $\omega$ be small as compared to the total kinetic energy of the quark, which is of the order of $E_{\mathrm{F}} \approx\left(\pi^{2} n_{b}\right)^{1 / 3}: \omega \ll\left(\pi^{2} n_{b}\right)^{1 / 3}$. Since at the star's surface the kinetic energy of the quarks is of the order of $300 \mathrm{MeV}$, the radiation formulae are valid only for frequencies $\omega \ll 300$ $\mathrm{MeV}$. In order to find the maximum frequency $\omega_{\max }$ of the radiation in the ultrarelativistic case, we assume that it is of the order of $\omega_{\max } \sim 1 / \tau\left(1-v^{2}\right)$; that is, it is inversely proportional to the duration of the collision and to the square of the energy of the radiating particle (Landau \& Lifshitz 1975). If we assume that quarks have energies near the Fermi energy, then the corresponding velocities are the Fermi velocities,

$$
v_{\mathrm{F}}=\frac{\sqrt{3 \pi / 4 \alpha_{s}}}{\sqrt{1+3 \pi / 4 \alpha_{s}}},
$$

which are of the order of $v_{\mathrm{F}}=0.92$ for $\alpha_{s}=0.4$ and $v_{\mathrm{F}}=0.85$ for $\alpha_{s}=0.9$. Hence, the maximum allowable frequency up to which the formalism can be applied is given by

$$
\omega_{\max } \sim \frac{1+3 \pi / 4 \alpha_{s}}{\tau} \sim n_{b} \sigma_{0} \sqrt{\frac{3 \pi}{4 \alpha_{s}}\left(1+\frac{3 \pi}{4 \alpha_{s}}\right)} .
$$

For quark-quark interaction cross sections of the order of 1 mbarn and for $\alpha_{s}=0.4$, we obtain $\omega_{\max } \sim 30 \mathrm{MeV}$. In the following we restrict our study to only quark bremsstrahlung frequencies satisfying the condition $\omega<\omega_{\max } \approx$ $30 \mathrm{MeV}$.

The frequency distribution of the radiation emitted by a single quark moving in the dense matter at the surface of a quark star is presented in Figure 3. The total intensity $I$ of the bremsstrahlung radiation emitted by a single quark,

$$
I=\frac{\alpha}{\pi} \sum_{l=u, d, s}\left(\frac{q_{l}}{e}\right)^{2} \int_{\omega_{\mathrm{LPM}}}^{\omega_{\max }} \frac{1}{v_{l}} \Phi\left(\omega, v_{l}, \tau^{-1}\right) \frac{d \omega}{\omega},
$$

obtained by integrating equation (13) over all possible frequencies, is represented as a function of the collision time $\tau$ in Figure 4.

For the same range of frequencies, the calculation of the bremsstrahlung intensity by using equation (15), which does not take into account the effects of the multiple collisions, 


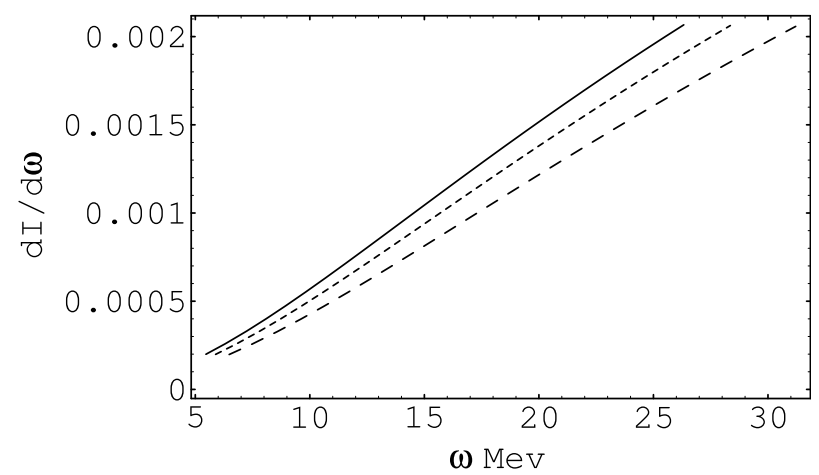

FIG. 3.-Frequency distribution $d I / d \omega$ of the bremsstrahlung radiation emitted due to quark-quark collisions at the surface of the strange star, for different values of the surface density: $n_{b}=0.24$ (solid curve), 0.30 (dotted curve), and $0.4 \mathrm{fm}^{-3}$ (dashed curve). In all cases we have considered a temperature of $T=5 \mathrm{MeV}$ for the strange matter. For the QCD coupling constant, we have chosen the value $\alpha_{s}=0.4$.

gives $I \sim 0.11 \mathrm{MeV}$. By taking into account that in the highdensity QGP the collision time is finite and of the order of $\tau \sim 10 \mathrm{fm}$ leads to an intensity of the order of $I \sim 0.03$ $\mathrm{MeV}$. Hence, the LPM effect could reduce the intensity of the photon bremsstrahlung of quarks at the surface of the star by a factor of $3-5$ for $n_{b} \in(0.24,0.4) \mathrm{fm}^{-3}$. For collision times of the order of $\tau \sim 5 \mathrm{fm}$, which, because of the uncertainties in the quark-quark cross sections, are also possible, the decrease in the intensity of the bremsstrahlung is of an order of magnitude. The variation of the intensity of the electromagnetic radiation is represented, as a function of the quark density and for different values of the temperature, in Figure 5.

The intensity of the radiation emitted by a single particle is decreasing with increasing density, because of the effects of the multiple scatterings of the quarks. The number of photons is proportional to the number of collisions, but if these happen too often, because of the high density of the medium, the photons emitted at different points of the trajectory start to interfere with each other, and the intensity of the radiation is accordingly reduced. On the other hand, $I$ increases with the temperature of the dense quark matter.

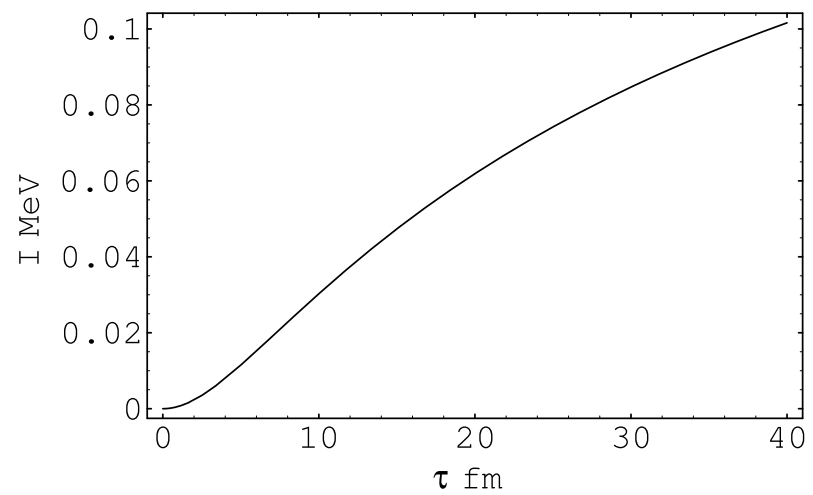

FIG. 4.-Variation of the total intensity $I$ of the bremsstrahlung radiation emitted by a single quark as a function of the collision time $\tau$. We assumed that the velocity of the quarks is the Fermi velocity and the QCD coupling constant is $\alpha_{s}=0.4$.

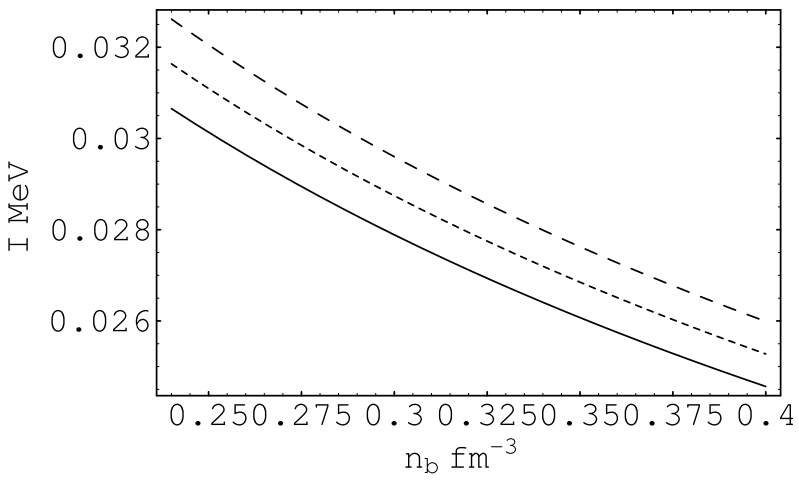

FIG. 5.-Total intensity $I$ of the bremsstrahlung radiation emitted by a single quark as a function of the strange star's surface density $n_{b} \mathrm{fm}^{-3}$, for different values of the temperature: $T=0.10$ (solid curve), 5 (dotted curve), and $10 \mathrm{MeV}$ (dashed curve). In all cases we have taken $\alpha_{s}=0.4$

If the quarks are extremely relativistic, with $v_{l} \rightarrow 1, l=u$, $d, s$, the intensity of the total radiation emitted by a single quark can be expressed in the following closed form:

$$
\begin{aligned}
\frac{\pi}{\alpha} I\left(n_{b}, T\right) \approx & \frac{1+3 \pi / 4 \alpha_{s}}{\tau} \ln \left[\frac{1+4\left(1+3 \pi / 4 \alpha_{s}\right)^{2}}{\left(1+4 \tau^{2} a^{2} n_{b}^{2 / 3}\right)^{a n_{b}^{1 / 3}}}\right] \\
& +\frac{1}{\tau}\left[\arctan 2\left(1+\frac{3 \pi}{4 \alpha_{s}}\right)-\arctan 2 \tau a n_{b}^{1 / 3}\right] \\
& -4\left(\frac{1+3 \pi / 4 \alpha_{s}}{\tau}-a n_{b}^{1 / 3}\right) \\
& +\frac{1}{2 \tau}\left[D\left(1+\frac{3 \pi}{4 \alpha_{s}}\right)-D\left(\tau a n_{b}^{1 / 3}\right)\right]
\end{aligned}
$$

where the function $D(x)$ is given by $D(x)=$ $i\left[\operatorname{Li}_{2}(-2 i x)-\mathrm{Li}_{2}(2 i x)\right]$. The function $D(x)$ is real for all $x$, and its power series expansion is given by $D(x)=$ $4 x-16 x^{3} / 9+64 x^{5} / 25-256 x^{7} / 49+O\left(x^{8}\right)$. The approximation $v \approx 1$ is appropriate for quarks having energies near the Fermi energy and moving with velocities $v(0)=v_{\mathrm{F}}$, which are of the order of 1 .

The emission of a photon in quark matter is the result of the scattering of a quark from a state $\boldsymbol{p}_{1}$ to a state $\boldsymbol{p}_{1}^{\prime}$ by collision with another particle of momentum $\boldsymbol{p}_{2}$. The rate of collisions in the QGP in which a soft photon of energy $\omega \ll E$ is emitted can be defined as (Chiu 1968)

$$
\begin{aligned}
d N= & g_{1} g_{2} f_{1}\left(\boldsymbol{p}_{1}\right) f_{2}\left(\boldsymbol{p}_{2}\right) \sigma(\theta, \phi)\left|\boldsymbol{v}_{1}-\boldsymbol{v}_{2}\right|\left[1-f_{1}\left(\boldsymbol{p}_{1}^{\prime}\right)\right] \\
& \times\left[1-f_{2}\left(\boldsymbol{p}_{2}^{\prime}\right)\right] \prod_{i=1,2,1^{\prime}, 2^{\prime}} d^{3} \boldsymbol{p}_{i} d \Omega(\theta, \phi),
\end{aligned}
$$

where $d \Omega(\theta, \phi)$ is the solid angle element in the direction $(\theta$, $\phi), g_{1}$ and $g_{2}$ are the statistical weights, and $\boldsymbol{v}_{1}$ and $\boldsymbol{v}_{2}$ are the velocities of the interacting particles. The bremsstrahlung emissivity per unit volume of the quark matter is given by

$$
\epsilon_{\mathrm{Br}}=\frac{d E_{\mathrm{Br}}}{d t d V}=\int \omega d N
$$

With the use of the explicit form of the cross section for the photon emission, given by equation (18), the photon emissivity can be written in the form

$$
\epsilon_{\mathrm{Br}}=\chi_{\mathrm{Br}}\left(n_{b}, T\right) T^{4},
$$


where $\chi_{\mathrm{Br}}$ is a function of the quark density and of the temperature.

The bremsstrahlung energy flux from the star $F_{\mathrm{Br}}$ is given by the emissivity of a thin surface layer, with thickness of the order of the photon mean free path $\lambda$ (Chmaj et al. 1991). Taking into account only photons emitted outward and produced not deeper than $\lambda$, the energy flux from the surface of the quark star is given by

$$
F_{\mathrm{Br}}=\sigma_{\mathrm{Br}}\left(n_{b}, T\right) T^{4}
$$

where $\sigma_{\mathrm{Br}}\left(n_{b}, T\right)=\chi_{\mathrm{Br}} \lambda / \pi$. Since the temperature dependence of $\sigma_{\mathrm{Br}}$ is not significant, it follows that the quark bremsstrahlung spectrum has the same temperature dependence as blackbody radiation.

For arbitrary temperatures the coefficient of the bremsstrahlung emissivity can be represented, in an approximate analytical form, by

$$
\sigma_{\mathrm{Br}}\left(n_{b}, T\right) \approx \frac{g^{2}}{(2 \pi)^{3}} \mathrm{Li}_{2}^{2}\left\{-\exp \left[\frac{\left(\pi^{2} n_{b}\right)^{1 / 3}}{T}\right]\right\} I\left(n_{b}, T\right) \lambda .
$$

The calculation of $\epsilon_{\mathrm{Br}}$ requires, in the general case, the estimation of integrals of the form $F=\int_{0}^{\infty} p d p /$ $\{[\exp (p-\mu) / T]+1\}$, which can be expressed usually in terms of a polylogarithmic function. In the limit of small temperatures, with $T \ll E_{\mathrm{F}}=\left(\pi^{2} n_{b}\right)^{1 / 3}$, the integral can be evaluated by means of the substitution $z=(p-\mu) / T$. For small $T$, one can extend the lower limit of integration to $-\infty$, so that the integral becomes $F=\int_{-\infty}^{+\infty} T(T z+\mu) d z$ / $[\exp (z)+1]=\left(\pi^{2} / 6\right) T^{2}$. Therefore, in the limit of small temperatures, $\epsilon_{\mathrm{Br}}=\sigma_{\mathrm{Br}}\left(n_{b}, T\right) T^{4}$, but there is no explicit temperature dependence of the coefficient $\sigma_{\mathrm{Br}}$ :

$$
\sigma_{\mathrm{Br}}\left(n_{b}, T\right) \approx \frac{g^{2} \pi}{576} I\left(n_{b}, T\right) \lambda .
$$

In this case the only $T$-dependence of $\sigma_{\mathrm{Br}}$ is via the intensity of the radiation.

The variation of the ratio of the bremsstrahlung emissivity coefficient and the Stefan-Boltzmann constant $\sigma_{\mathrm{SB}}$ is represented, as a function of the mean collision time $\tau$ and in the limit of small temperatures, in Figure 6. We have assumed that the thickness of the emitting layer is $\lambda=5 \mathrm{fm}$.

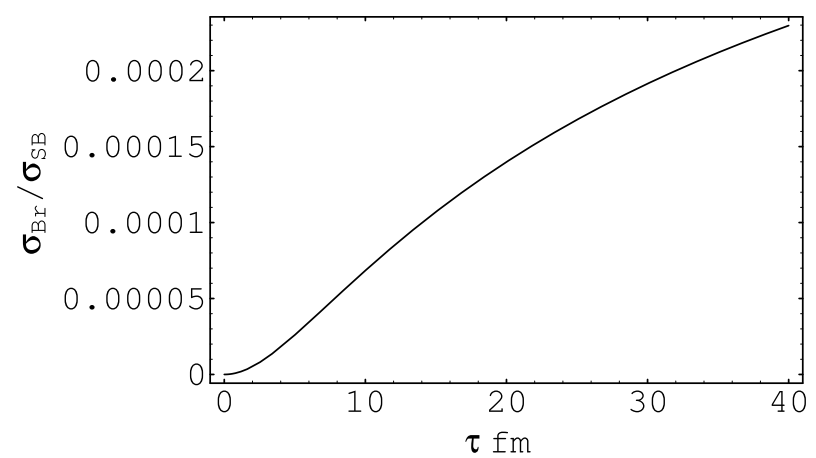

FIG. 6.-Variation of the ratio $\sigma_{\mathrm{Br}} / \sigma_{\mathrm{SB}}$ of the bremsstrahlung emissivity of the quark matter to the Stefan-Boltzmann constant, as a function of the mean collision time $\tau$ at the quark star surface. The thickness of the emitting quark layer is $\lambda=5 \mathrm{fm}$.
In the limit of large collision times, $\sigma_{\mathrm{Br}} / \sigma_{\mathrm{SB}} \sim 10^{-4}$, and thus we recover the single-collision results of Chmaj et al. (1991). For collision times specific to the QGP at the surface of the quark stars, of the order of $10 \mathrm{fm}$, the ratio $\sigma_{\mathrm{Br}} / \sigma_{\mathrm{SB}} \sim 10^{-5}$. Therefore, taking into account multiple collisions in the high-density plasma reduces by an order of magnitude the value of the bremsstrahlung coefficient $\sigma_{\mathrm{Br}}$, making the soft photon radiation from the surface of the quark star about 5 orders of magnitude weaker than equilibrium blackbody radiation.

\section{EFFECT OF THE ELECTRON LAYER ON THE PHOTON EMISSIVITY OF QUARK STARS}

The radiation emitted by the thin quark surface layer of the strange star must propagate through the electron layer formed due to the electrostatic attraction of the quarks. In the following, we consider the effect of the electron layer on the electromagnetic radiation emitted, via the bremsstrahlung mechanism, by the quarks in the layer of thickness $\lambda$. As a first step, it is necessary to obtain a quantitative analytical description of the properties of the electrons outside the quark star.

To find the electron distribution near the quark star surface, we follow the approach of Alcock et al. (1986) but also taking into account the finite temperature effects as discussed by Kettner et al. (1995). The chemical equilibrium implies that the electron chemical potential $\mu_{\infty}=-V+\mu_{e}$ is constant, where $V$ is the electrostatic potential per unit charge and $\mu_{e}$ is the electron's chemical potential. Since far outside the star both $V$ and $\mu_{e}$ tend to 0 , it follows that $\mu_{\infty}=0$ and $\mu_{e}=V$.

For the number density $n_{e}$ of the electrons at the quark star surface, we use the expression (Kettner et al. 1995)

$$
n_{e}(z, T)=\frac{1}{3 \pi^{2}} \mu_{e}^{3}+\frac{1}{3} \mu_{e} T^{2}=\frac{1}{3 \pi^{2}} V^{3}+\frac{1}{3} V T^{2},
$$

where $T$ is the temperature of the electron layer, which can be taken as a constant, since we assume the electrons are in thermodynamic equilibrium with the constant-temperature quark matter.

The Poisson equation for the electrostatic potential $V(z, T)$ generated by the finite temperature electron distribution reads (Alcock et al. 1986; Kettner et al. 1995)

$$
\begin{gathered}
\frac{d^{2} V}{d z^{2}}=\frac{4 \alpha}{3 \pi}\left[\left(V^{3}-V_{q}^{3}\right)+\pi^{2}\left(V-V_{q}\right) T^{2}\right], \quad z \leq 0, \\
\frac{d^{2} V}{d z^{2}}=\frac{4 \alpha}{3 \pi}\left(V^{3}+\pi^{2} V T^{2}\right), \quad z \geq 0 .
\end{gathered}
$$

In equations (34) and (35), $z$ is the space coordinate measuring height above the quark surface, $\alpha$ is the fine-structure constant, and $V_{q} / 3 \pi^{2}$ is the quark charge density inside the quark matter. The boundary conditions for equations (34) and (35) are $V \rightarrow V_{q}$ as $z \rightarrow-\infty$ and $V \rightarrow 0$ for $z \rightarrow \infty$. In the case of the zero-temperature electron distribution at the boundary $z=0$, we have the condition $V(0)=(3 / 4) V_{q}$ (Alcock et al. 1986).

In the following, we are interested mainly in the properties of the electron gas extending outside the quark surface of the star, described by equation (35). In order to find the exact solution of equation (35), we introduce first a new variable $y=d V / d z$. Then, equation (35) can be written in 
the form

$$
\frac{d y^{2}}{d V}=\frac{8 \alpha}{3 \pi}\left(V^{3}+\pi^{2} V T^{2}\right)
$$

immediately leading to the first integral

$$
\frac{d V}{d z}=\sqrt{\frac{4 \alpha}{3 \pi}} \sqrt{\frac{V^{4}}{2}+\pi^{2} V^{2} T^{2}},
$$

where an arbitrary integration constant has been set to 0 .

The general solution of equation (37) is given by

$$
V(z, T)=\frac{4 \sqrt{2} \pi T \exp \left[2 \sqrt{\alpha \pi / 3} T\left(z+z_{0}\right)\right]}{\exp \left[4 \sqrt{\alpha \pi / 3} T\left(z+z_{0}\right)\right]-1},
$$

where $z_{0}$ is a constant of integration. Its value can be obtained from the condition of the continuity of the potential across the star's surface, requiring $V_{I}(0, T)=V(0, T)$, where $V_{I}(z, T)$ is the value of the electrostatic potential in the region $z \leq 0$, described by equation (34). Therefore,

$$
z_{0}=\frac{1}{2} \sqrt{\frac{3}{\alpha \pi}} \frac{1}{T} \ln \left\{\frac{2 \sqrt{2} \pi T}{V_{I}(0, T)}\left[1+\sqrt{1+\frac{V_{I}^{2}(0, T)}{8 \pi^{2} T^{2}}}\right]\right\} .
$$

Hence, the electron number density $n_{e}(z, T)$ is given, at finite temperatures, by

$$
\begin{aligned}
& n_{e}(z, T)=\frac{4 \sqrt{2} \pi}{3} \frac{\exp \left[2 \sqrt{\alpha \pi / 3} T\left(z+z_{0}\right)\right]}{\exp \left[4 \sqrt{\alpha \pi / 3} T\left(z+z_{0}\right)\right]-1} \\
& \quad \times\left\{32\left(\frac{\exp \left[2 \sqrt{\alpha \pi / 3} T\left(z+z_{0}\right)\right]}{\exp \left[4 \sqrt{\alpha \pi / 3} T\left(z+z_{0}\right)\right]-1}\right)^{2}+1\right\} T^{3}, \quad T \neq 0 .
\end{aligned}
$$

In the limit of zero temperature, $T \rightarrow 0$, from equation (37) we obtain

$$
V(z)=\frac{a_{0}}{z+b},
$$

where $a_{0}=(3 \pi / 2 \alpha)^{1 / 2}$ and $b$ is an integration constant. Here $b$ can be determined from the boundary condition $V(0)=(3 / 4) V_{q}$, which gives $b=\left(4 a_{0} / 3 V_{q}\right)$. Therefore, in this case for the electron particle number distribution, extending several thousand fermis above the quark matter surface, we find the expression

$$
n_{e}(z)=\frac{1}{3 \pi^{2}} \frac{a_{0}^{3}}{(z+b)^{3}}, \quad T=0 .
$$

The variation of the electron number density $n_{e}(z, T)$ is represented, for different values of the temperature, in Figure 7.

In the absence of a crust of the quark star, the electron layer can extend to several thousand fermis outside the star's surface.

The intensity of the photon beam coming from the thin outer layer of the quark star decreases because of the energy absorption in the electron layer. The variation of the intensity of the beam can be obtained by solving the equation of radiative transfer (Shapiro \& Teukolsky 1983). For a steady

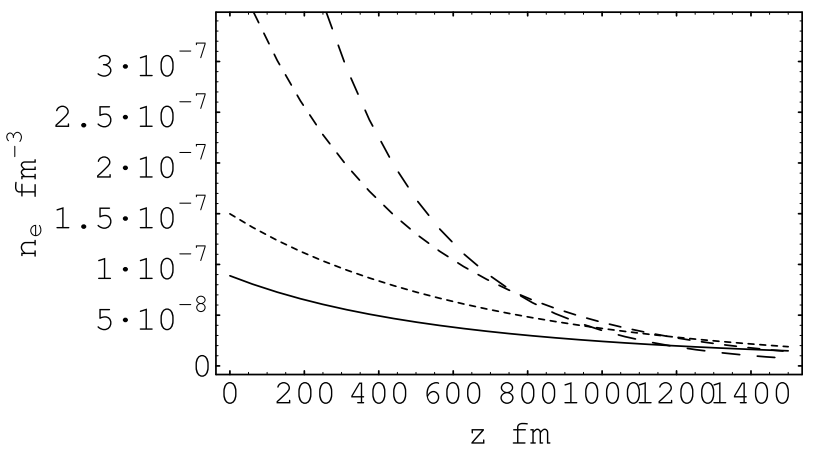

FIG. 7.-Variation of the electron number density $n_{e}$ as a function of the distance $z(\mathrm{fm})$ for different values of the temperature: $T=0$ (solid curve), 1.5 (dotted curve), 2.5 (short-dashed curve), and $3.5 \mathrm{MeV}$ (long-dashed curve). For the $T=0$ case we have chosen $V_{q}=20 \mathrm{MeV}$, while for $T \neq 0$, $V_{I}(0, T)=14 \mathrm{MeV}$

state, the general solution of the transport equation is given by

$$
I_{\omega}=I_{\omega}(0) e^{-\tau_{\omega}}+\int_{0}^{\tau_{\omega}} j_{\omega}(z, T) e^{-\left(\tau_{\omega}-\tau_{\omega}^{\prime}\right)} d \tau_{\omega}^{\prime}
$$

where the optical depth $\tau_{\omega}$ is defined as $d \tau_{\omega}=k_{\omega} \rho d z$, with $k_{\omega}$ the Rosseland mean opacity (giving the energy absorbed per unit time per unit volume from the beam with intensity $\left.I_{\omega}\right), I_{\omega}(0)$ is the intensity of the radiation incident on the electron layer, and $j_{\omega}(z, T)$ is the emissivity of the electron in the layer. Since in the present paper we are interested in studying only the effect of the outer electrons on the quark matter emission, we take $j_{\omega}(z, T) \approx 0$; that is, we neglect the emissivity of the electron layer. Hence, the variation of the intensity, due to absorption in the electron layer, of the photon beam emitted by the quarks at the strange star surface can be described by the equation

$$
I_{\omega}=I_{\omega}(0) e^{-\tau_{\omega}} .
$$

In the case of the scattering of photons by free nonrelativistic electrons, the opacity is given by $k_{\omega}=\sigma_{\mathrm{T}} n_{e}(z) / \rho$, where $\sigma_{\mathrm{T}}=(8 \pi / 3)\left(e^{2} / m_{e} c^{2}\right)^{2}$ is the Thomson cross section (Berestetskii et al. 1982; Rybicki \& Lightman 1979). This formula is valid only in the case of a nondegenerate gas at low temperatures. For systems at high densities and/or temperatures, the corrections to the Thomson scattering cross section become important.

For high-temperature nondegenerate electrons, the opacity was calculated first in Sampson (1959). These calculations have been extended to the semidegenerate $(T \neq 0)$ case by Chin (1965) and Buchler \& Yueh (1976; see also Boercker 1987 and Rose 1995). For a degenerate electron gas at $T \neq 0$, the Rosseland mean opacity is given by (Buchler \& Yueh 1976)

$$
k_{\omega}=\frac{n_{e} \sigma_{\mathrm{T}}}{\rho} G^{\operatorname{deg}}(T, \eta) .
$$

Here, $G^{\operatorname{deg}}(T, \eta)$ (the inverse of the Rosseland mean) is a function of the temperature and of the degeneracy parameter $\eta=\left(E_{\mathrm{F}}-m_{e} c^{2}\right) / k T$, where $E_{\mathrm{F}}$ is the Fermi energy. 


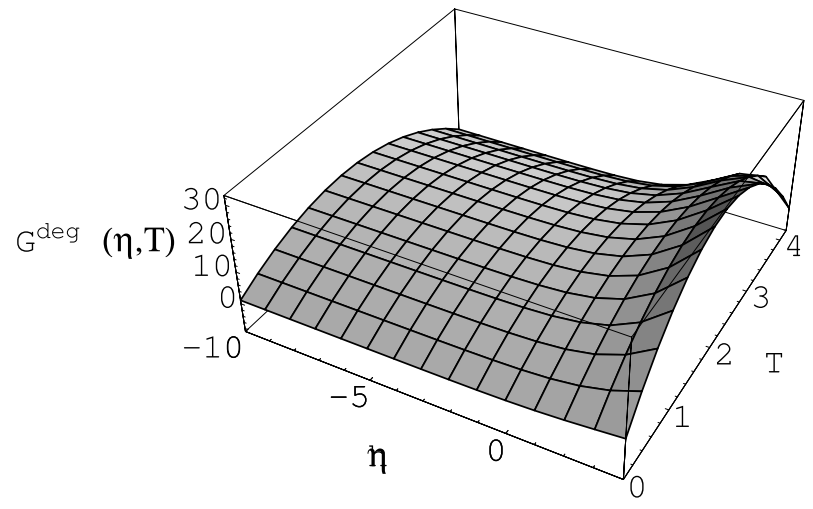

FIG. 8.-Variation of the function $G^{\operatorname{deg}}(T, \eta)$ for $\eta \in(-10,+4)$ and $T \in\left(0,4.5 m_{e} c^{2}\right)$

The function $G^{\operatorname{deg}}(T, \eta)$ can be represented in the form (Buchler \& Yueh 1976)

$$
\begin{aligned}
G^{\operatorname{deg}}(T, \eta)= & 1.129+0.2965 \xi-0.005594 \xi^{2} \\
& +\left(11.47+0.3570 \xi+0.1078 \xi^{2}\right) T \\
& +\left(-3.249+0.1678 \xi-0.04706 \xi^{2}\right) T^{2},
\end{aligned}
$$

where $\xi=\exp \left(0.8168 \eta-0.05522 \eta^{2}\right)$. The variation of the function $G^{\mathrm{deg}}$ as a function of the parameters $\eta$ and $T$ is represented in Figure 8.

For a nondegenerate electron gas obeying the MaxwellBoltzmann distribution, the Rosseland mean opacity is given by (Sampson 1959)

$$
\begin{aligned}
G^{\text {ndeg }}(u, T)=1 & +2 T+5 T^{2}+\frac{15}{4} T^{3} \\
& -\frac{1}{5}\left(16+103 T+408 T^{2}\right)(u T) \\
& +\left(\frac{21}{2}+\frac{609}{5} T\right)(u T)^{2}-\frac{2203}{70}(u T)^{3},
\end{aligned}
$$

where $u=h \omega / k T$ and the temperature is expressed in units of $m_{e} c^{2}$

In order to obtain a simple estimate of the absorption of the surface radiation of quarks due to the presence of the electron layer, we consider first the case of the completely degenerate electron gas at a small temperature $T$. Complete degeneracy corresponds to $\eta \rightarrow-\infty$ and $\xi \rightarrow 0$. We also neglect the temperature-dependent terms in equation (46), which are not important for small temperatures. Hence, from equation (46) we obtain, as a first approximation, $G^{\operatorname{deg}}(0,-\infty) \approx 1.129$.

Therefore, the Rosseland opacity of the electron layer at the quark star surface can be approximated, for low temperatures of the degenerate electron gas, by the expression

$$
\begin{aligned}
k_{\omega}(z) & =\frac{n_{e} \sigma_{\mathrm{T}} G^{\mathrm{deg}}(0,-\infty)}{\rho} \\
& =\frac{1}{3 \pi^{2}} \frac{G^{\mathrm{deg}}(0,-\infty) \sigma_{\mathrm{T}}}{\rho} \frac{a_{0}^{3}}{(z+b)^{3}} .
\end{aligned}
$$

Consequently, for the optical depth of the electron distribution above the quark star surface, we find the expression

$$
\begin{aligned}
\tau_{\omega} & =\frac{1}{3 \pi^{2}} G^{\mathrm{deg}}(0,-\infty) \sigma_{\mathrm{T}} a_{0}^{3} \int_{0}^{z} \frac{d z}{(z+b)^{3}} \\
& =\frac{1}{6 \pi^{2}} G^{\operatorname{deg}}(0,-\infty) \sigma_{\mathrm{T}} a_{0}^{3}\left[\frac{1}{b^{2}}-\frac{1}{(z+b)^{2}}\right] .
\end{aligned}
$$

For large $z$, corresponding to the limit $z \rightarrow \infty$, from equation (49) we obtain

$$
\begin{aligned}
\tau_{\omega} & =\frac{1}{6 \pi^{2}} G^{\mathrm{deg}}(0,-\infty) \sigma_{\mathrm{T}} \frac{a_{0}^{3}}{b^{2}} \\
& =\frac{3}{32 \pi^{3 / 2}} V_{q}^{2} \sqrt{\frac{3}{2 \alpha}} G^{\operatorname{deg}}(0,-\infty) \sigma_{\mathrm{T}} .
\end{aligned}
$$

At zero temperature the optical depth is proportional to the square of the value of the quark charge density at the surface of the quark star $V_{q}$.

The absorption of the bremsstrahlung radiation by the electron layer essentially depends on the quark charge density $V_{q}=(1 / 3)\left(2 n_{u}-n_{d}-n_{s}\right)$ inside the quark star, where $u, d$, and $s$ denote the different quark flavors (Cheng et al. 1998a). The condition of charge neutrality requires $V_{q}^{3}=n_{e}^{(\text {int) }}$, where $n_{e}^{\text {(int) }}$ is the charge density of the electrons inside the star and is related to the baryon number density $n_{b}=\left(n_{u}+n_{d}+n_{s}\right) / 3$ by means of the relation $n_{e}^{\text {(int) }}=Y_{e} n_{b}$, where for the electron abundance $Y_{e}$ we adopt the range $Y_{e} \in\left(10^{-5}, 10^{-3}\right)$ (Cheng et al. 1998a). Near the surface of the quark star, the density can be approximated by $\rho \approx 4 B \approx 4 \times 10^{14} \mathrm{~g} \mathrm{~cm}^{-3}$. For a strange quark mass of the order of $m_{s} \approx 300 \mathrm{MeV}$, for the baryon number density near the surface of the star we find $n_{b} \approx 7.68 \times 10^{6} \mathrm{MeV}^{3}$. Hence, the corresponding quark charge density is $V_{q}=197 Y_{e}^{1 / 3} \mathrm{MeV}$, leading to $V_{q} \in(4,20) \mathrm{MeV}$.

The corresponding bremsstrahlung luminosity $L^{\mathrm{qs}}$ of the quark star is given by $L^{\mathrm{qs}}=\eta_{\mathrm{eff}} 4 \pi R^{2} L_{\mathrm{Br}}=\eta_{\mathrm{eff}} 4 \pi R^{2} \sigma_{\mathrm{Br}} T^{4}$, where $R$ is the radius of the star. For a $T=0$ star and for a degenerate electron layer,

$$
\begin{aligned}
\eta_{\text {eff }} & =\exp \left(-\tau_{\omega}\right) \\
& =\exp \left[-\left(\frac{3}{32} \pi^{3 / 2}\right) V_{q}^{2}(0) \sqrt{\frac{3}{2 \alpha}} G^{\operatorname{deg}}(0,-\infty) \sigma_{\mathrm{T}}\right] .
\end{aligned}
$$

The variation of the coefficient $\eta_{\text {eff }}$ is represented, as a function of $V_{q}$, in Figure 9.

For an increasing surface quark charge density, $\eta_{\text {eff }}$ is a rapidly decreasing function. Therefore, in the case of a large $V_{q}$, the electron layer significantly decreases the intensity of the bremsstrahlung radiation emitted by the thin quark layer at the surface of the star.

The spectrum of the $T \neq 0$ quark star essentially depends on the charge density $V_{I}(0, T)$ at the surface of the star, on the degeneracy parameter $\eta$, and on the temperature. The numerical values of $V_{I}(0, T)$ must be obtained by solving equation (34) in the region $z \leq 0$ for different values of $T$, together with the boundary condition $V \rightarrow V_{q}$ for $z \rightarrow-\infty$. Therefore, even for the nonzero-temperature quark star, the spectrum of the bremsstrahlung radiation is determined by the quark charge density inside the star. However, in order to obtain a simple estimate of the temperature effects on the optical length of the radiation in the electron layer, we 


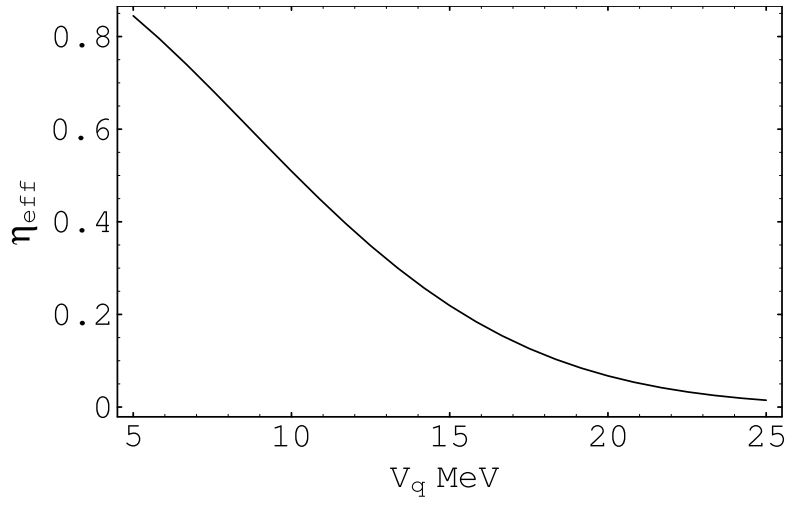

FIG. 9.-Variation of $\eta_{\text {eff }}$ as a function of the strange quark surface potential $V_{q}$.

assume that for a suitable range of temperatures, the charge density at the surface can be approximated by $V_{I}(0, T) \approx 14 \mathrm{MeV}$ (Alcock et al. 1986; Kettner et al. 1995). For this value of the charge density, the variation of $\eta_{\text {eff }}$ as a function of $\eta$ and $T$ is represented in Figure 10.

Since the increase in the temperature also increases the electron number density of the electron layer, the absorption of the bremsstrahlung radiation is much stronger at high temperatures of the semidegenerate electron gas.

\section{DISCUSSIONS AND FINAL REMARKS}

Since the proposal of the existence of strange stars, much effort has been devoted to finding observational properties that could distinguish strange stars from neutron stars. One of the most common and simplest methods could be to find some specific signatures in the photon emissivity of strange stars. In the first estimate of the surface photon emissivity of quark matter (Chmaj et al. 1991), it was concluded that the most important photon radiation mechanism is the bremsstrahlung of

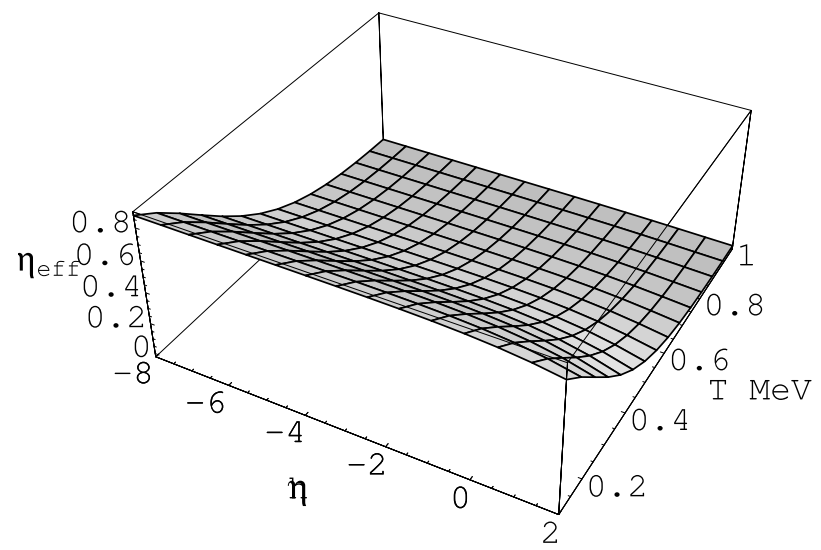

FIG. 10.-Variation of $\eta_{\text {eff }}$ as a function of the degeneracy parameter $\eta$ and of the temperature $T$ for the case of a partially degenerate electron gas, for a quark surface charge density potential $V_{I}=14 \mathrm{MeV}$. quarks from a thin layer at the star's surface and that the intensity of the radiation is about 4 orders of magnitude weaker than the equilibrium blackbody radiation, although both have the same temperature dependence.

In the present paper we have reconsidered the electromagnetic emission from the surface of bare quark stars by also taking into account the important effect of the multiple collisions of quarks in the very high density QGP existing at the surface of the quark stars. To obtain the bremsstrahlung spectrum of the photon emission of the quarks, we have used several assumptions. The most important is the adoption of a specific model for the description of quark-quark elastic scattering, which is essential in obtaining the bremsstrahlung emissivity. The details of the interactions between quarks are generally poorly known, and therefore there are a lot of uncertainties in the description of the strong interactions in a QGP. In our calculations we have used a classical soft photon approximation, neglecting quantum effects. We have generally assumed that the quarks are semidegenerate, with temperature $T>0$ and $T \ll E_{\mathrm{F}}$ but with the kinetic energies of the interacting particles of the order of the Fermi energy.

In order to give a complete description of the quarkquark bremsstrahlung, we have considered, in a systematic way, the effects of the dense medium on the radiation spectrum. A dense medium in which many collisions occur will reduce the bremsstrahlung radiation, and the higher the density is, the lower will be the bremsstrahlung emission. On the other hand, the emission of photons with frequency smaller than the critical LPM frequency is completely suppressed. Here $\omega_{\text {LPM }}$ depends on the QCD coupling constant and on the density at the quark star's surface. For strange stars, $\omega_{\text {LPM }}$ is of the order of $\omega_{\text {LPM }} \approx 5-6 \mathrm{MeV}$, so photons with $\omega<\omega_{\text {LPM }}$ cannot be emitted from the surface of the star. Hence, only low frequencies are suppressed because of the LPM effect. On the other hand, the multiple collision effect reduces by 1 order of magnitude the bremsstrahlung coefficient $\sigma_{\mathrm{Br}}$, making the soft photon radiation from the quark star 5 orders of magnitude weaker than the blackbody radiation.

A second effect, which essentially influences the radiation of photons by quark stars, is the absorption of the radiation in the electron layer formed outside the quark star. The electron layer could extend to several hundred or thousand fermis, the electron number density being an increasing function of the temperature. The high-density semidegenerate electron layer at the surface of a strange star plays an important role in the propagation of the electromagnetic radiation from the star's surface. The optical depth of the degenerate or semidegenerate electron layer depends on the electron number density, temperature, degeneracy parameter, and charge density at the surface of the quark star. For high surface charge densities, the intensity of the quark bremsstrahlung radiation is significantly reduced by absorption in the electron layer. For a low-temperature star, with $T \ll E_{\mathrm{F}}$, for a surface potential $V_{q} \approx 20 \mathrm{MeV}$, the electron layer reduces the intensity of the radiation by an order of magnitude. In this case the combined effects of the multiple collisions and absorption in the electron layer give $\sigma_{\mathrm{Br}} / \sigma_{\mathrm{SB}} \sim 10^{-6}$. Hence, the energy radiated away by the quark star could be 6 orders of magnitude smaller than equilibrium blackbody radiation.

For the sake of comparison we also present the expression for the photon emission rate of an equilibrated, hot QCD 
plasma, at zero chemical potential, with the LPM effect fully included. The spontaneous emission rate $(2 \pi)^{3} d I_{\gamma} / d^{3} k$ of photons with a given momentum $\boldsymbol{k}$ can be represented, for two-flavor QCD, as (Arnold et al. 2001, 2002)

$(2 \pi)^{3} \frac{d I_{\gamma}}{d^{3} k}=\frac{40 \pi \alpha \alpha_{s}}{9} T^{2} \frac{n_{f}(\omega)}{\omega}\left[\ln \frac{T}{m_{\infty}}+C_{\text {tot }}\left(\frac{\omega}{T}\right)\right]$,

where $n_{f}(\omega)$ is the Fermi-Dirac distribution function, $n_{f}(\omega)=[\exp (\omega / T)+1]^{-1}, m_{\infty}$ is the thermal quark mass, given by $m_{\infty}^{2}=4 \pi \alpha_{s} T^{2} / 3$, and

$$
\begin{aligned}
C_{\text {tot }}\left(\frac{\omega}{T}\right) \equiv & \frac{1}{2} \ln \frac{2 \omega}{T}+C_{2 \leftrightarrow 2}\left(\frac{\omega}{T}\right)+C_{\mathrm{Br}}\left(\frac{\omega}{T}\right) \\
& +C_{\text {annih }}\left(\frac{\omega}{T}\right),
\end{aligned}
$$

with

$$
\begin{aligned}
C_{2 \leftrightarrow 2}(x) & \approx 0.041 x^{-1}-0.3615+1.01 \exp (-1.35 x), \\
C_{\mathrm{Br}}(x)+C_{\mathrm{annih}}(x)=\left(1+\frac{N_{f}}{6}\right)^{1 / 2} & \\
\times & {\left[\frac{0.548 \ln (12.28+1 / x)}{x^{3 / 2}}+\frac{0.133 x}{(1+x / 16.27)^{1 / 2}}\right] . }
\end{aligned}
$$

For $\omega / T<2.5$, bremsstrahlung becomes the most important process, while for $\omega / T>10$, pair annihilation dominates. The LPM effect suppresses both bremsstrahlung and pair annihilation processes, but the suppression is not severe ( $35 \%$ or less), except for photons with $\omega<2 T$ or very hard pair annihilation.

In the limit of small temperatures, $T \rightarrow 0$ and $x \rightarrow \infty$. Hence,

$$
\begin{gathered}
C_{2 \leftrightarrow 2}(x) \rightarrow-0.3615, \\
C_{\mathrm{Br}}(x)+C_{\mathrm{annih}}(x) \rightarrow 0.133\left(\frac{\omega}{T}\right)^{1 / 2} .
\end{gathered}
$$

By neglecting the logarithmically divergent terms, the integration over all possible ranges of frequencies in equation (52) gives

$$
I_{\gamma} \sim \chi_{\mathrm{Br}}^{(\mathrm{QCD})} T^{4}
$$

where

$$
\begin{aligned}
\chi_{\mathrm{Br}}^{(\mathrm{QCD})} \approx & 5 \pi \alpha \alpha_{s}\left[3\left(1+N_{f} / 6\right)^{1 / 2}\right. \\
& \left.\times(4-\sqrt{2})^{1 / 2} \pi^{-3 / 2} \xi(5 / 2) / 4-0.3615 / 3\right] / 9
\end{aligned}
$$

and $\xi(s)$ is the Riemann zeta function $\xi(s)=\sum_{k=1}^{\infty} k^{-s}$. Therefore, the perturbative QCD approach gives, in the low-temperature limit, the same temperature dependence of the bremsstrahlung spectrum as in equation (29). However, we must point out that equations (52) and (53) have been derived for a temperature of the QGP higher than $250 \mathrm{MeV}$, since the temperature of the fireball formed in heavy-ion collisions is of the order of 450 MeV (Renk 2003).

Recently, it has been argued that the strange quark matter in the color-flavor locked (CFL) phase of QCD, which occurs for large gaps $(\Delta \sim 100 \mathrm{MeV})$, is rigorously electrically neutral, despite the unequal quark masses, and even in the presence of an electron chemical potential (Alford, Rajagopal, \& Wilczek 1998, 1999; Rajagopal \& Wilczek 2001). However, Page \& Usov (2002) pointed out that for sufficiently large $m_{s}$, the low-density regime is rather expected to be in the "two-color-flavor superconductor" phase, in which only $u$ and $d$ quarks of two colors are paired in single condensates, while the ones of the third color and $s$ quarks of all three colors are unpaired. In this phase, electrons are present. In other words, electrons may be absent in the core of strange stars but present, at least, near the surface where the density is lowest. Nevertheless, the presence of the CFL effect can reduce the electron density at the surface and hence increase the bremsstrahlung emissivity.

In conclusion, in the present paper we have pointed out two effects that could significantly reduce or even fully suppress the bremsstrahlung radiation from the surface of quark stars: the significant decrease of the intensity due to the multiple collisions in a dense medium and the absorption by the outer electron layer of the stars. The possible observational significance for the detection of quark stars of these effects will be considered in a future publication.

The authors would like to thank the anonymous referee, whose comments helped to improve the manuscript. We are grateful for the useful comments of Professor V. V. Usov. This work was supported by the Research Grants Council of the Hong Kong Government.
Aggarwal, M. M., et al. 2000, Phys. Rev. Lett., 85, 3595 Alcock, C., Farhi, E., \& Olinto, A. 1986, ApJ, 310, 261 Alford, M., Rajagopal, K., \& Wilczek, F. 1998, Phys. Lett. B, 422, 247 . 1999, Nucl. Phys. B, 537, 443

Anthony, P. L., et al. 1995, Phys. Rev. Lett., 75, 1949

Arnold, P., Moore, G. D., \& Yaffe, L. G. 2001, J. High Energy Phys., 12, 9 2002, J. High Energy Phys., 6, 030

Aurenche, P., Gelis, F., \& Zaraket, H. 2000, Phys. Rev. D, 62, 096012

Baier, R., Nakkagawa, H., Niegawa, A., \& Redlich, K. 1992, Z. Phys. C, 53,433

Berestetskii, V. B., Lifshitz, E. M., \& Pitaevskii, L. P. 1982, Quantum Electrodynamics (Oxford: Pergamon)

Boercker, D. B. 1987, ApJ, 316, L95

Buchler, J. R., \& Yueh, W. R. 1976, ApJ, 210, 440

Cheng, K. S., \& Dai, Z. G. 1996, Phys. Rev. Lett., 77, 1210 1998, Phys. Rev. Lett., 80, 18

Cheng, K. S., Dai, Z. G., \& Lu, T. 1998a, Int. J. Mod. Phys. D, 7, 139

Cheng, K. S., Dai, Z. G., Wei, D. M., \& Lu, T. 1998b, Science, 280, 407

\section{REFERENCES}

Cheng, K. S., \& Harko, T. 2000, Phys. Rev. D, 62, 083001

Chin, C. W. 1965, ApJ, 142, 1481

Chiu, H.-Y. 1968, Stellar Physics (Waltham: Blaisdell)

Chmaj, T., Haensel, P., \& Slominski, W. 1991, Nucl. Phys. B, 24, 40

Cleymans, J., Goloviznin, V. V., \& Redlich, K. 1993, Phys. Rev. D, 47, 173

Dai, Z. G., Peng, Q. H., \& Lu, T. 1995, ApJ, 440, 815

Dey, M., Bombacci, I., Dey, J., Ray, S., \& Samanta, B. C. 1998, Phys. Lett. B, 438,123

Gondek-Rosinska, D., Bulik, T., Zdunik, L., Gourgoulhon, E., Ray, S., Dey, J., \& Dey, M. 2000, A\&A, 363, 1005

Gourgoulhon, E., Haensel, P., Livine, R., Paluch, E., Bonazzola, S., \& Marck, J.-A. 1999, A\&A, 349, 851

Haensel, P., Zdunik, J. L., \& Schaeffer, R. 1986, A\&A, 160, 121

Haglin, K., \& Pratt, S. 1994, Phys. Lett. B, 328, 255

Harko, T., \& Cheng, K. S. 2002, A\&A, 385, 947

Hatsuda, T., \& Kunihiro, T. 1994, Phys. Rep., 247, 221

Jackson, J. D. 1999, Classical Electrodynamics (New York: Wiley)

Kapusta, J., Lichard, P., \& Seibert, D. 1991, Phys. Rev. D, 44, 2774 
Kettner, Ch., Weber, F., Weigel, M. K., \& Glendenning, N. K. 1995, Phys. Rev. D, 51, 1440

Klein, S. 1999, Rev. Mod. Phys., 71, 1501

Landau, L. D., \& Lifshitz, E. M. 1975, The Classical Theory of Fields (Oxford: Pergamon)

Landau, L. D., \& Pomeranchuk, I. J. 1953, Dokl. Akad. Nauk. SSSR, 92, 735

Manley, D. M. 1997, Phys. Rev. D, 56, 7325

Page, D., \& Usov, V. V. 2002, Phys. Rev. Lett., 89, 131101

Peitzmann, T., \& Thoma, M. H. 2002, Phys. Rep., 364, 175

Rajagopal, K., \& Wilczek, F. 2001, Phys. Rev. Lett., 86, 3492

Renk, T. 2003, Phys. Rev. C, 67, 064901
Rose, S. J. 1995, ApJ, 453, L45

Rybicki, G. B., \& Lightman, A. P. 1979, Radiative Processes in Astrophysics (New York: Wiley)

Sampson, D. H. 1959, ApJ, 129, 734

Shapiro, S. L., \& Teukolsky, S. A. 1983, Black Holes, White Dwarfs, and Neutron Stars (New York: Wiley)

Usov, V. V. 1998, Phys. Rev. Lett., 80, 230

Witten, E. 1984, Phys. Rev. D, 30, 272

Zdunik, J. L. 2000, A\&A, 359, 311

Zhuang, P., Hufner, J., Klevansky, S. P., \& Neise, L. 1995, Phys. Rev. D, 51, 3728 